\title{
Evaluation of Nematicides and Oil Cakes against Root Knot Nematode caused by Meloidogyne incognita in Pomegranate
}

\author{
Madhushri Kerakalamatti ${ }^{1 *}$, R. K. Mesta $^{1}$, K. C. Kiran Kumar ${ }^{2}$ and D. L. Rudresh ${ }^{3}$ \\ ${ }^{1}$ Department of Plant Pathology, College of Horticulture Bagalkote, Karnataka, India \\ ${ }^{2}$ Horticulture Research and Extension Center, Arsikere, Hassan, Karnataka, India \\ ${ }^{3}$ Department of Agricultural Microbiology, College of Horticulture Bagalkote, \\ Karnataka, India \\ *Corresponding author
}

\section{A B S T R A C T}

\section{Keywords}

Management,

Meloidogyne

incognita,

Nematicide, Oil

cake, Pomegranate,

Root knot nematode

Article Info

Accepted:

17 June 2020

Available Online:

10 July 2020
Pomegranate (Punica granatum L.) is one of the important fruit crops belongs to the family Lythraceae. Nowadays most of the fields were infested by root knot nematode caused by Meloidogyne incognita which results in yellowing and stunting of plant further it affects the total yield of the crop. Four nematicides and five oil cakes were evaluated against number of juveniles emerged from egg mass at 24, 48 and 72 hours interval against $M$. incognita in laboratory condition for their efficacy against the pathogen. Further evaluated before and after inoculation of pathogen in potsto check the effect onplant growth parameters like fresh shoot weight, dry shoot weight, fresh root weight, dry root weight, shoot length, root length and disease parameters like yellowing of leaves and number of galls (grade) in all the treatments in comparison to uninoculated control. Among the nematicides phorate $(0.01 \mathrm{~g} / \mathrm{ml})$ was found effective as it recorded significantly least numbers over all other nematicides with respect to number of juveniles emerged (1.56) followed by carbosulfan (11.22). Among the oil cakes neem cake was found significantly superior over all other nematicides as it recorded least number of juveniles emerged (2.45) followed by pongamia cake (7.33). The number of juveniles emerged increased steadily as number of hours increased. Among the treatments, neem cake and phorate applied plants prior and after inoculation of $M$. incognita showed higher fresh shoot weight, dry shoot weight, fresh root weight, dry root weight, shoot length, root length and less number of galls.

\section{Introduction}

Pomegranate (Punica granatum L.) is an attractive, highly prized, nutrient rich fruit and is a longlived drought tolerant plant. Arid and semiarid zones are popular for growing pomegranate trees. Pomegranate belongs to the family Lythreceae, having $2 n=16$ number of chromosome and it is native to Iran. The basket of pomegranate was chosen as a symbol of plenty for the $18^{\text {th }}$ International Horticultural Congress, held in 1970. However, in the recent past pomegranate cultivation has been highly threatened due to 
incidence of root knot nematode (Meloidogyne incognita) infestation. The estimation of yield losses due to root knot nematode, in pomegranate has been reported to the extent of 17.3 per cent (Jain et al., 2010).

Root knot nematode infested plants shows above ground symptoms like yellowing of leaves normally produced, occasionally some plants revealed stunting and wilt symptoms by senescing the entire plant's foliage at once. In the belowground small to large round root galls were found which were white in colour turned to light brown and hardy when they became old. Pinkish to red colour egg masses were observed inside as well as outside of the galls which contain two hundred fifty to thousands of juveniles. More number of females were found in a single compound gall. Severe infection resulted in dying of whole tree causing severe yield losses leading to death of affected plants in a few weeks (Plate 1).

The oil cakes and nematicides are important components for the management of nematodes. Therefore, an effort is needed in this regard to see the efficacy of some oil cakes and nematicides against root knot causing nematode both in laboratory and pot condition before going to field management.

\section{Materials and Methods}

\section{In vitro evaluation of nematicides against} M. incognita

The nematicides tested are given in Table A.

Above mentioned nematicides at required concentrations were added to $90 \mathrm{~mm}$ diameter sterilized Petri plate containing $20 \mathrm{ml}$ of distilled water. For each petri plate five female egg masses of Meloidogyne incognita were inoculated. The number of juveniles emerged were counted with needle at $24 \mathrm{~h}$, 48h and 72h intervals using stereomicroscope.

In vitro evaluation of oil cakes against $M$. incognita

Details of the oil cakes are given in Table B

Above mentioned oil cakes $(2 \mathrm{~g}$ in powdered form) were added to $90 \mathrm{~mm}$ diameter sterilized petri plate containing $20 \mathrm{ml}$ of distilled water. For each petri plate five female egg masses of Meloidogyne incognita were inoculated. The number of juveniles emerged were counted with needle at $24 \mathrm{~h}$, $48 \mathrm{~h}$ and $72 \mathrm{~h}$ intervals using stereomicroscope.

Efficacy of nematicides and oil cakes against $M$. incognita under pot culture

Above mentioned nematicides and oil cakes at required concentrations were added before and one week after inoculation of (1000 $\mathrm{J}_{2}$ stage) $M$. incognita juveniles to pomegranate plant. Observations like disease and plant growth parameters were checked after 60 days of inoculation.

\section{Results and Discussion}

\section{In vitro evaluation of nematicides against M. incognita}

Four nematicides were evaluated against number of juveniles emerged at 24, 48 and 72 hours interval against $M$. incognita in laboratory condition for their efficacy against the pathogen as described in "Material and Methods". The results are presented in the Table 1, Fig. 1.

Among the nematicides phorate $(0.01 \mathrm{~g} / \mathrm{ml})$ was found effective as it recorded significantly least numbers over all other nematicides with respect to number of juveniles emerged (1.56) followed by carbosulfan (11.22), carbofuran (11.33). Least inhibiting capacity was found in cartap 
hydrochloride (17.22) over the control.

The number of juveniles emerged increased steadily as number of hours increased. However, the increase was very less in phorate @ $0.01 \% / \mathrm{ml}(1.33,1.67$ and 1.63$)$ for juveniles emerged. This was followed by carbosulfan@ $0.2 \mu 1 / \mathrm{ml}(4.33,14.33$ and 15.00). The control recorded (10.67, 15.67 and 25.67) for juveniles emerged.

In vitro evaluation of oil cakes against $M$. incognita

Five oil cakes were tested against number of juveniles emerged at 24, 48 and 72 hours interval against $M$. incognita in laboratory condition for their efficacy against the pathogen as described in "Material and Methods". The results are presented in the Table 2, Fig. 2.

Among the oil cakes neem cake was found significantly superior over all other nematicides as it recorded least number of juveniles emerged (2.45) followed by pongamia cake (7.33), mustard cake (12.33), and jatropha cake (18.45) while the control recorded 26.00 .

The number of juveniles emerged increased steadily as number of hours increased. However, the increase was very less in neem cake @ 0.01\%/ml (0.00, 1.67 and 5.97) followed by pongamia cake @ $0.2 \mu \mathrm{l} / \mathrm{ml}$ (6.00, 7.67 and 8.33) for juveniles emerged. The control recorded (17.00, 27.00 and 34.00) for juveniles emerged.

\section{Efficacy of nematicides and oil cakes against $M$. incognita under pot culture}

Four nematicides and five Oil cakes were tested against $M$. incognita before and after inoculation of pathogen in potsto check the effect onplant growth parameters like fresh shoot weight, dry shoot weight, fresh root weight, dry root weight, shoot length, root length and disease parameters like yellowing of leaves and number of galls (grade) in all the treatments in comparison to uninoculated control. Data presented in Table 3, Plate 2a \& $2 \mathrm{~b}$ and $3 \mathrm{a} \& 3 \mathrm{~b}$.

Among the treatments, neem cake and phorate applied plants prior and after inoculation of $M$. incognita showed higher fresh shoot weight, dry shoot weight, fresh root weight, dry root weight, shoot length, root length and less number of galls (Plate 4 and 5).

Application of phorate @ $9 \mathrm{~g} / \mathrm{pot}$ and neem cake @ $50 \mathrm{~g} /$ pot before the inoculation of $M$. incognita did not recorded any galls. Treatment of phorate one week prior to inoculation of $M$. incognita plants recorded maximum number of fresh shoot weight of $(103.0 \mathrm{~g})$, dry shoot weight $(71.6 \mathrm{~g})$, fresh root weight (30.0 g), dry root weight $(19.1 \mathrm{~g})$, shoot length $(95.0 \mathrm{~cm})$ and root length $(9.5$ $\mathrm{cm})$ found compared to other treatment. Treatment of neem cake one week after inoculation of $M$. incognita plants with maximum number of fresh shoot weight of $(98.0 \mathrm{~g})$, dry shoot weight $(61.4 \mathrm{~g})$, fresh root weight $(32.0 \mathrm{~g})$, dry root weight $(23.5 \mathrm{~g})$, shoot length $(99.0 \mathrm{~cm})$ and root length $(9.5$ $\mathrm{cm})$. In both the treatments plants were healthy.

Treatment of neem cake one week prior to inoculation of $M$. incognita plants had high fresh shoot weight of (98.6 g), dry shoot weight $(68.7 \mathrm{~g})$, fresh root weight $(29.0 \mathrm{~g})$, dry root weight $(18.5 \mathrm{~g})$, shoot length $(92.0$ $\mathrm{cm})$, root length $(9.5 \mathrm{~cm})$ and no galls found compared to control. And treatment of neem cake one week after inoculation of $M$. incognita plants also had high fresh shoot weight of $(102.0 \mathrm{~g})$, dry shoot weight $(68.0$ $\mathrm{g})$, fresh root weight $(28.0 \mathrm{~g})$, dry root weight $(21.1 \mathrm{~g})$, shoot length $(96.0 \mathrm{~cm})$, root length $(9.8 \mathrm{~cm})$ and there are no galls formed compared to control. In both the treatments plants were healthy. 
There is high shoot and root weight was observed in castor cake treated plants. But the efficacy of castor cake was low compares to neem cake and phorate in case of nematode management.

Pomegranate (Punica granatum L.) has been rated as an important cash crop among horticulture crops. This crop is prone to several diseases, among which root knot cosed by Meloidogyne incognita is a one of the potential threats to successful crop production. Protection of crop plants from disease causing agents has been the focal point of the scientists in dealing with plant pathogens. Prohibitive costs of chemicals and their adverse ecological impact has made diversions of research priorities from chemical methods to other alternatives.
Hence, both nematicides and oil cakes which have nematicide property had evaluated against $M$. incognita in causing root knot infestation in pomegranate.

Nematicides were tested against number of juveniles emerged from the egg mass against $M$. incognita in laboratory condition for their efficacy against the pathogen. Among the nematicides phorate $(0.01 \mathrm{~g} / \mathrm{ml})$ was found significantly superior over all other nematicides. Phorate is an organophosphate and carbosulfan is a carbamate group chemical, which mainly effect on nervous system of nematodes by inhibiting acetylcholinesterase (AChE) enzyme a chemical messenger that function as neurotransmitter.

Table.A Tested nematicides

\begin{tabular}{|c|l|l|l|c|}
\hline $\begin{array}{c}\text { S. } \\
\text { No. }\end{array}$ & Name of the chemical & \multicolumn{1}{|c|}{ Chemical name } & $\begin{array}{c}\text { Trade } \\
\text { name }\end{array}$ & $\begin{array}{c}\text { Dosage g/ Kg of } \\
\text { soil }\end{array}$ \\
\hline $\mathbf{1 .}$ & Aldicarb 15G & $\begin{array}{l}\text { 2-Methyl-2-(methylthio) propanal } \\
\text { o- (N-methylcarbamoyl) oxime }\end{array}$ & Temik & 0.5 \\
\hline $\mathbf{2 .}$ & Carbosulfan 25E & $\begin{array}{l}\text { 2, 2-Dimethyl-2, 3-dihydro-1- } \\
\text { benzofuran-7-yl [ (dibutylamino) } \\
\text { sulfanyl] methylcarbamate }\end{array}$ & Marshal & 0.75 \\
\hline 3. & $\begin{array}{l}\text { Cartap hydrochloride } \\
\text { 50\% SP }\end{array}$ & $\begin{array}{l}\text { S, S'-[-2 (dimethylamino) } \\
\text { trimethylene]-bis (thiocarbamate) } \\
\text { hydrochloride }\end{array}$ & Cartap & 0.5 \\
\hline $\mathbf{4 .}$ & Phorate 10\% CG & $\begin{array}{l}\text { 0, 0-diethyl-S (ethyl thiomethyl) } \\
\text { dithiophophate }\end{array}$ & Thimet & 0.45 \\
\hline
\end{tabular}

Table.B Details of the oil cakes

\begin{tabular}{|c|l|l|}
\hline Sl. No. & \multicolumn{1}{|c|}{ Oil cakes } \\
\hline $\mathbf{1 .}$ & Castor & Botanical name \\
\hline $\mathbf{2 .}$ & Jatropha & Jatropha communis \\
\hline $\mathbf{3 .}$ & Mustard & Brasssica juncea \\
\hline $\mathbf{4 .}$ & Neem & Azadirachta indica \\
\hline $\mathbf{5 .}$ & Pongamia & Pongamia pinnata \\
\hline
\end{tabular}


Table.1 In vitro evaluation of nematicides against Meloidogyne incognita

\begin{tabular}{|c|c|c|c|c|c|}
\hline \multirow{2}{*}{$\begin{array}{l}\text { Sl. } \\
\text { No. }\end{array}$} & \multirow[t]{2}{*}{ Nematicide } & \multicolumn{4}{|c|}{ Number of juveniles emerged } \\
\hline & & $\begin{array}{c}\text { @24 } \\
\text { hours }\end{array}$ & $\begin{array}{c}@ 48 \\
\text { hours }\end{array}$ & $\begin{array}{c}\text { @72 } \\
\text { hours }\end{array}$ & Mean \\
\hline 1 & Carbofuran $(0.02 \mathrm{~g} / \mathrm{ml})$ & $\begin{array}{c}4.00 \\
(2.23)\end{array}$ & $\begin{array}{c}7.33 \\
(2.89)\end{array}$ & $\begin{array}{l}22.67 \\
(4.86)\end{array}$ & $\begin{array}{l}11.33 \\
(3.33)\end{array}$ \\
\hline 2 & Carbosulfan $(0.2 \mu \mathrm{l} / \mathrm{ml})$ & $\begin{array}{c}4.33 \\
(2.31)\end{array}$ & $\begin{array}{l}14.33 \\
(3.91)\end{array}$ & $\begin{array}{l}15.00 \\
(3.99)\end{array}$ & $\begin{array}{l}11.22 \\
(3.40)\end{array}$ \\
\hline 3 & $\begin{array}{l}\text { Cartap hydrochloride } \\
(0.1 \mathrm{mg} / \mathrm{ml})\end{array}$ & $\begin{array}{l}10.33 \\
(3.37)\end{array}$ & $\begin{array}{l}16.67 \\
(4.20)\end{array}$ & $\begin{array}{l}24.67 \\
(5.07)\end{array}$ & $\begin{array}{l}17.22 \\
(4.21)\end{array}$ \\
\hline 4 & Phorate $(0.01 \mathrm{~g} / \mathrm{ml})$ & $\begin{array}{c}1.33 \\
(1.52)\end{array}$ & $\begin{array}{c}1.67 \\
(1.63)\end{array}$ & $\begin{array}{l}1.67 \\
(1.63)\end{array}$ & $\begin{array}{c}1.56 \\
(1.59)\end{array}$ \\
\hline 5 & Control & $\begin{array}{l}10.67 \\
(3.42)\end{array}$ & $\begin{array}{l}15.67 \\
(4.08)\end{array}$ & $\begin{array}{l}25.67 \\
(5.16)\end{array}$ & $\begin{array}{l}17.33 \\
(4.22)\end{array}$ \\
\hline & Mean & $\begin{array}{c}6.13 \\
(2.57)\end{array}$ & $\begin{array}{l}11.13 \\
(3.34)\end{array}$ & $\begin{array}{l}17.93 \\
(4.14)\end{array}$ & \\
\hline & Source & \multicolumn{2}{|c|}{ 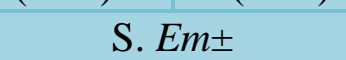 } & \multicolumn{2}{|c|}{ CD@1\% } \\
\hline & Nematicide & \multicolumn{2}{|c|}{0.07} & \multicolumn{2}{|c|}{0.20} \\
\hline & Hour & \multicolumn{2}{|c|}{0.05} & \multicolumn{2}{|c|}{0.15} \\
\hline & Nematicide X Hour & \multicolumn{2}{|c|}{0.12} & \multicolumn{2}{|c|}{0.34} \\
\hline
\end{tabular}

$*$ Values in parenthesis are $\sqrt{\mathrm{X}+1}$ transformed values

Table.2 In vitro evaluation of organic oil cakes against Meloidogyne incognita

\begin{tabular}{|c|c|c|c|c|c|}
\hline \multirow[t]{2}{*}{ Sl. No. } & \multirow[t]{2}{*}{ Nematicide } & \multicolumn{4}{|c|}{ Number of juveniles emerged } \\
\hline & & @ 24 hours & @ 48 hours & @72 hours & Mean \\
\hline 1 & Castor cake $(0.1 \mathrm{~g} / \mathrm{ml})$ & $\begin{array}{l}10.33 \\
(3.37)\end{array}$ & $\begin{array}{l}14.00 \\
(3.87)\end{array}$ & $\begin{array}{l}23.00 \\
(4.89)\end{array}$ & $\begin{array}{l}15.78 \\
(4.04)\end{array}$ \\
\hline 2 & Jatropha cake $(0.1 \mathrm{~g} / \mathrm{ml}))$ & $\begin{array}{c}5.67 \\
(2.55)\end{array}$ & $\begin{array}{l}20.67 \\
(4.59)\end{array}$ & $\begin{array}{l}29.00 \\
(5.47)\end{array}$ & $\begin{array}{l}18.45 \\
(4.20)\end{array}$ \\
\hline 3 & Mustard cake $(0.1 \mathrm{~g} / \mathrm{ml})$ & $\begin{array}{c}7.00 \\
(2.83)\end{array}$ & $\begin{array}{l}12.33 \\
(3.64)\end{array}$ & $\begin{array}{l}17.67 \\
(4.32)\end{array}$ & $\begin{array}{l}12.33 \\
(3.60)\end{array}$ \\
\hline 4 & Neem cake $(0.1 \mathrm{~g} / \mathrm{ml})$ & $\begin{array}{c}0.00 \\
(1.00)\end{array}$ & $\begin{array}{c}1.67 \\
(1.48)\end{array}$ & $\begin{array}{l}5.67 \\
(2.58\end{array}$ & $\begin{array}{c}2.45 \\
(1.24)\end{array}$ \\
\hline 5 & Pongamia cake $(0.1 \mathrm{~g} / \mathrm{ml})$ & $\begin{array}{c}6.00 \\
(2.64)\end{array}$ & $\begin{array}{c}7.67 \\
(2.94)\end{array}$ & $\begin{array}{c}8.33 \\
(3.05)\end{array}$ & $\begin{array}{c}7.33 \\
(2.88)\end{array}$ \\
\hline 6 & Control & $\begin{array}{l}17.00 \\
(4.24)\end{array}$ & $\begin{array}{l}27.00 \\
(5.29)\end{array}$ & $\begin{array}{l}34.00 \\
(5.92)\end{array}$ & $\begin{array}{l}26.00 \\
(5.15)\end{array}$ \\
\hline & Mean & $\begin{array}{c}7.67 \\
(2.77)\end{array}$ & $\begin{array}{l}13.89 \\
(3.64)\end{array}$ & $\begin{array}{l}19.61 \\
(4.37)\end{array}$ & \\
\hline & Source & \multicolumn{2}{|c|}{ S. $E m \pm$} & \multicolumn{2}{|c|}{ CD@1\% } \\
\hline & Organic amendment & \multicolumn{2}{|c|}{0.12} & \multicolumn{2}{|c|}{0.36} \\
\hline & Time & \multicolumn{2}{|c|}{0.09} & \multicolumn{2}{|c|}{0.25} \\
\hline \multicolumn{2}{|c|}{ Organic amendment X Hour } & \multicolumn{2}{|c|}{0.22} & \multicolumn{2}{|c|}{0.62} \\
\hline
\end{tabular}

*Values in parenthesis are $\sqrt{\mathrm{X}+1}$ transformed values 


\section{Int.J.Curr.Microbiol.App.Sci (2020) 9(7): xx-Xx}

Table.3 Evaluation of nematicides and oil cakes against Meloidogyne incognita under pot condition

\begin{tabular}{|c|c|c|c|c|c|c|c|c|c|c|c|c|c|c|c|c|c|c|}
\hline \multirow{3}{*}{$\begin{array}{l}\text { Sl. } \\
\text { No. }\end{array}$} & \multirow{3}{*}{\multicolumn{2}{|c|}{ *Treatment }} & \multicolumn{8}{|c|}{ Prior application of nematicides and oil cakes } & \multicolumn{8}{|c|}{ Later application of nematicides and oil cakes } \\
\hline & & & \multicolumn{2}{|c|}{$\begin{array}{c}\text { Disease } \\
\text { parameters }\end{array}$} & \multicolumn{6}{|c|}{ Plant growth parameters } & \multicolumn{2}{|c|}{$\begin{array}{c}\text { Disease } \\
\text { parameters }\end{array}$} & \multicolumn{6}{|c|}{ Plant growth parameters } \\
\hline & & & 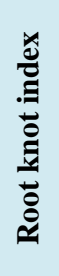 & 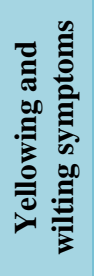 & 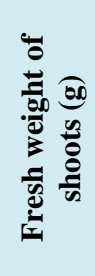 & 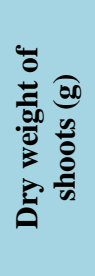 & 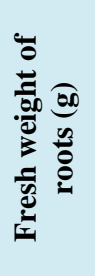 & 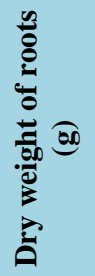 & 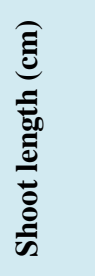 & 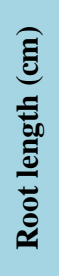 & 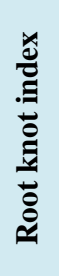 & 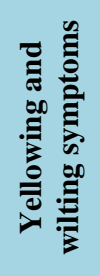 & 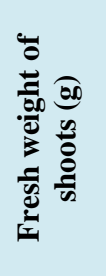 & 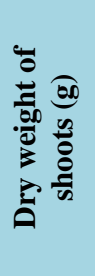 & 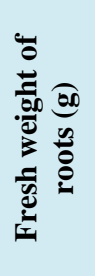 & 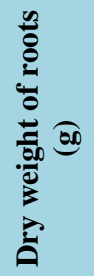 & 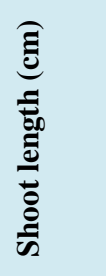 & 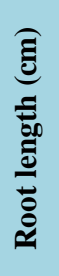 \\
\hline 1 & \multirow{4}{*}{ 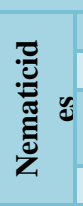 } & Carbofuran $(10 \mathrm{~g} / \mathrm{pot})$ & 3 & _ & 80.0 & 58.7 & 25.0 & 16.6 & 96.0 & 7.0 & 5 & - & 73.0 & 44.0 & 22.0 & 23.2 & 78.0 & 7.8 \\
\hline 2 & & Carbosulfan (15 g/pot) & 1 & + & 34.0 & 24.4 & 27.0 & 15.4 & 66.0 & 7.2 & 1 & - & 62.0 & 38.30 & 28.0 & 19.8 & 76.0 & 9.0 \\
\hline 3 & & $\begin{array}{l}\text { Cartap hydrochloride (10 } \\
\text { g/pot) }\end{array}$ & 2 & + & 28.5 & 15.2 & 10.0 & 10.6 & 73.0 & 8.3 & 1 & + & 21.0 & 17.1 & 24.0 & 10.0 & 87.0 & 8.5 \\
\hline 4 & & Phorate (9 g/pot) & 0 & _- & 103.0 & 71.6 & 30.0 & 19.1 & 95.0 & 9.5 & 0 & - & 98.0 & 61.4 & 32.0 & 23.5 & 99.0 & 9.5 \\
\hline 5 & \multirow{6}{*}{ 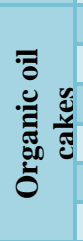 } & Castor cake (50 g/pot) & 2 & + & 77.0 & 26.3 & 14.0 & 6.0 & 122.0 & 8.0 & 1 & - & 104.0 & 45.0 & 42.0 & 38.7 & 140.0 & 7.9 \\
\hline 6 & & Jatropha cake (50 g/pot) & 4 & _ & 50.0 & 29.1 & 23.0 & 13.1 & 97.0 & 7.9 & 3 & + & 32.0 & 19.1 & 12.0 & 19.8 & 75.0 & 8.3 \\
\hline 7 & & Mustard cake (50 g/pot) & 2 & + & 70.0 & 48.5 & 19.0 & 10.5 & 120.0 & 7. & 1 & + & 93.0 & 61.3 & 25.0 & 19.2 & 90.0 & 8.0 \\
\hline 8 & & Neem cake $(50 \mathrm{~g} / \mathrm{pot})$ & 0 & _- & 98.6 & 68.7 & 29.0 & 18.5 & 92.0 & 9.5 & 0 & - & 102.0 & 68.0 & 28.0 & 21.1 & 96.0 & 9.8 \\
\hline 9 & & Pongamia cake $(50 \mathrm{~g} / \mathrm{pot})$ & 2 & - & 70.0 & 37.6 & 14.0 & 17.1 & 60.0 & 8.7 & 2 & + & 52.0 & 27.7 & 16.0 & 11.3 & 92.0 & 8.7 \\
\hline 10 & & Untreated control & 4 & + & 26.5 & 13.3 & 12.5 & 7.16 & 65.0 & 5.2 & 4 & + & 26.5 & 13.3 & 12.5 & 7.1 & 65.0 & 5.2 \\
\hline
\end{tabular}

*Each pot contains $20 \mathrm{~kg}$ sterilized soil. 


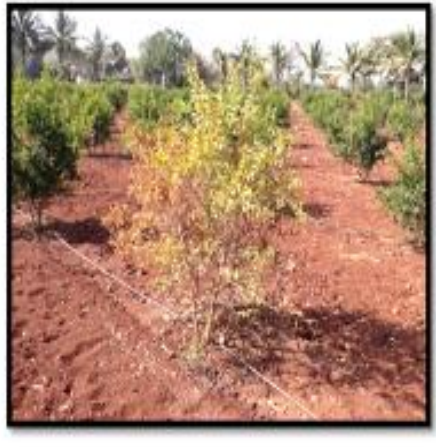

Yellowing of pomegranate leaves

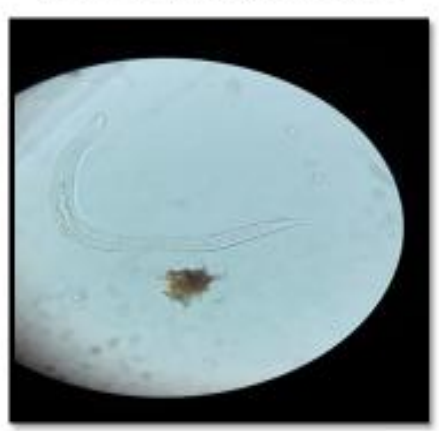

Male root knot nematode

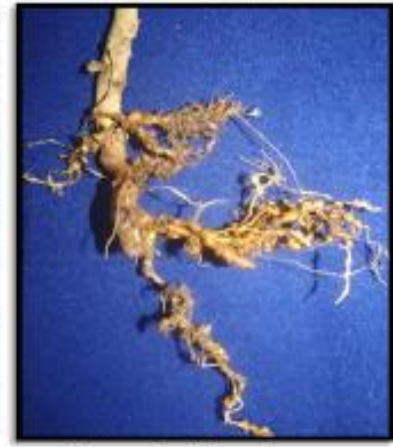

Nematode infestation on pomegranate roots

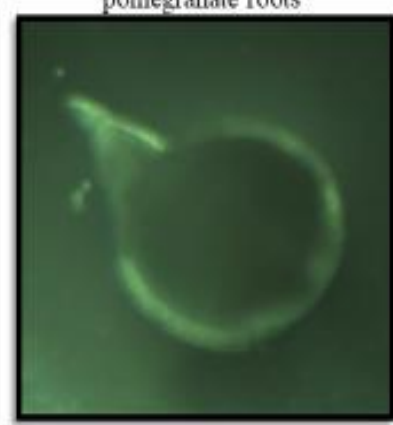

Female root knot nematode

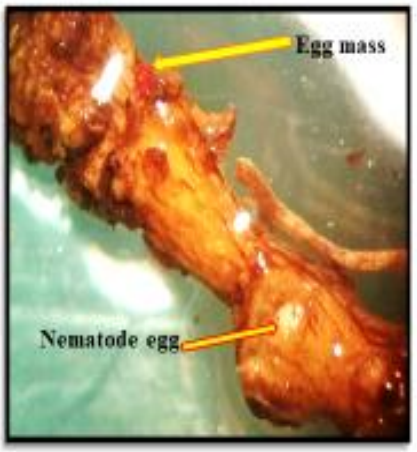

Root knot nematode eggs and egg mass

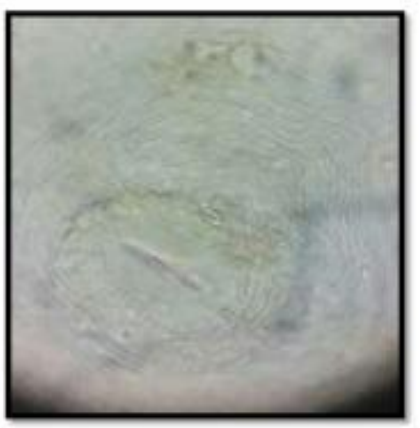

Perineal pattern of root knot nematode-Meloidogyne incognita

Plate 1: Pomegranate above and below ground symptoms and morphology of Root knot Nematode - Meloidogyne incognita

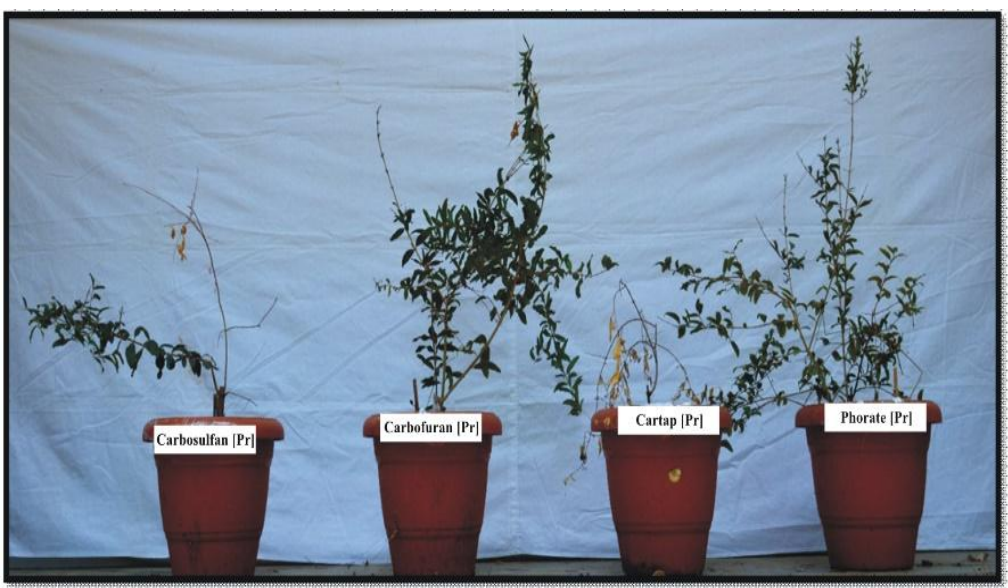

a) Effect of one week prior application of nematicides on development of symptoms on pomegranate seedlings caused by root knot nematodes

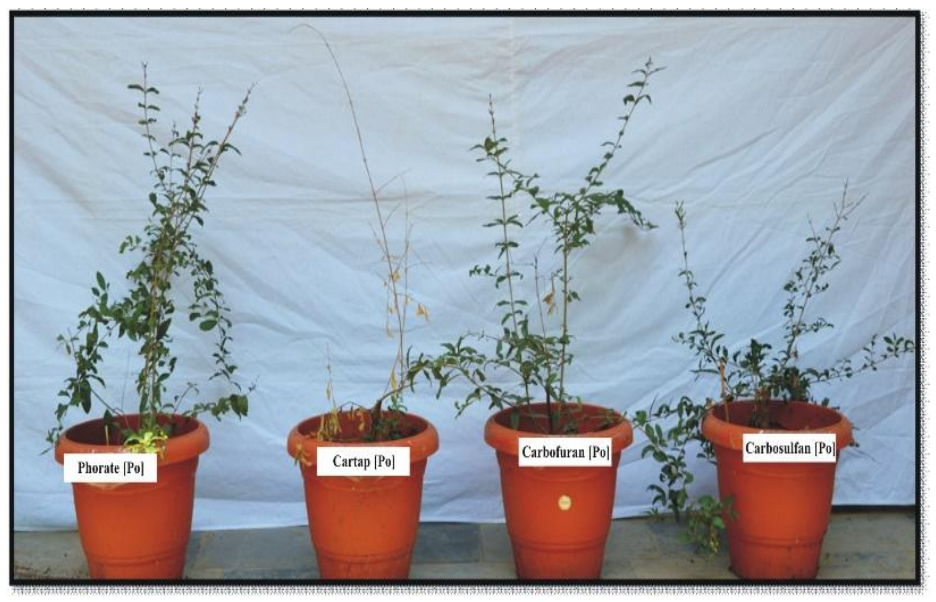

b) Effect of one week later application of nematicides on development of symptoms on pomegranate seedlings caused by root knot nematodes

Plate.2 Effect of nematicides on disease and plant growth parameter 


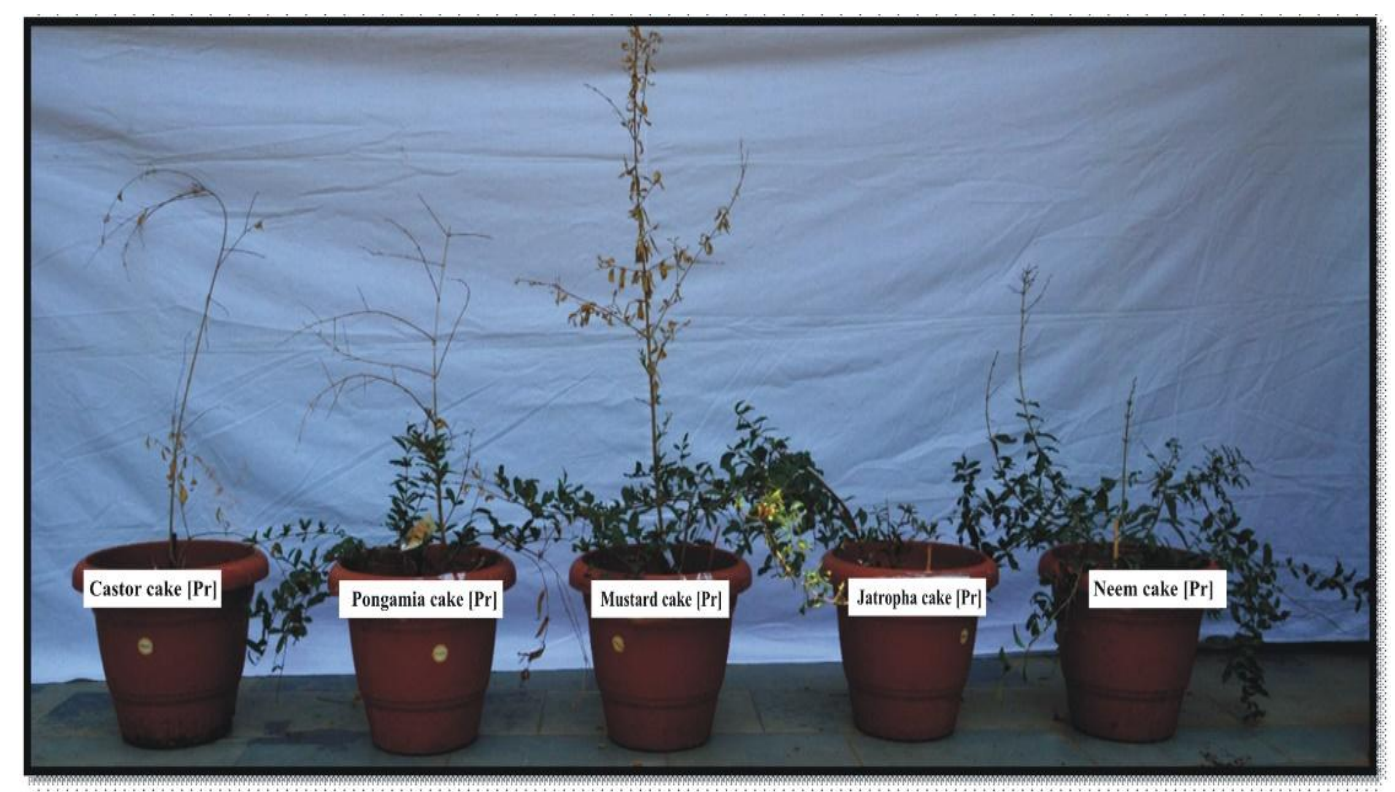

a) Effect of one week prior application of oil cakes on development of symptoms on pomegranate seedlings caused by root knot nematodes

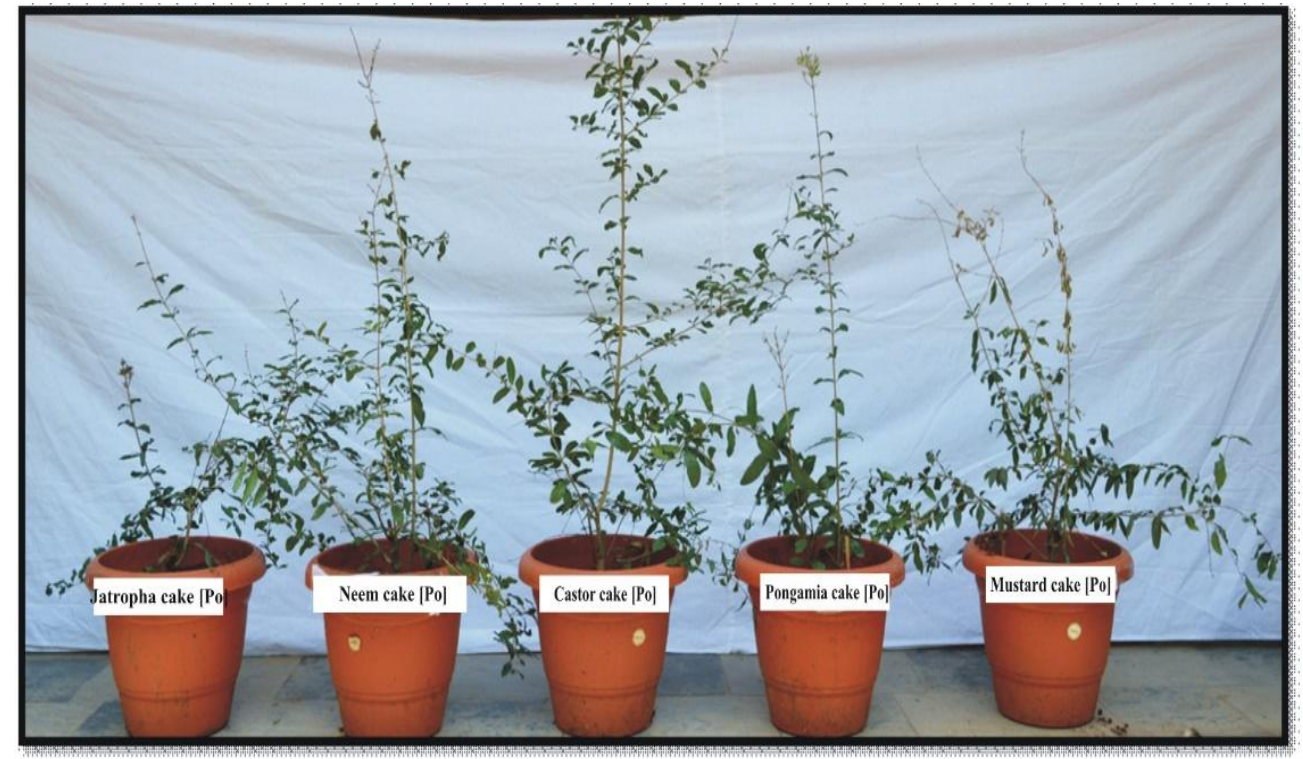

b) Effect of one week later application of oil cakes on development of symptoms on pomegranate seedlings caused by root knot nematodes

Plate.3 Effect of oil cakes on disease and plant growth parameter of pomegranate 

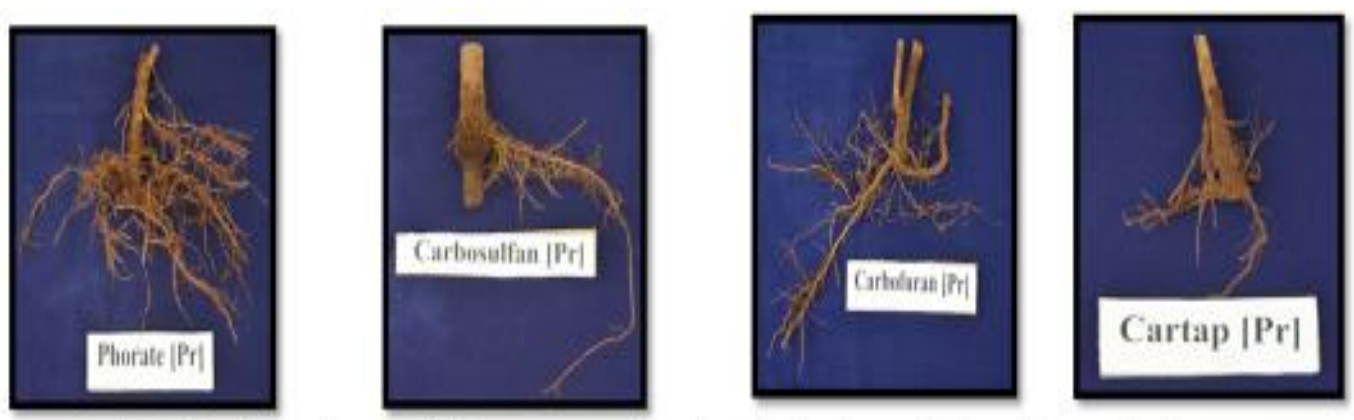

Application of nematicides one week prior to the inoculation of nematodes
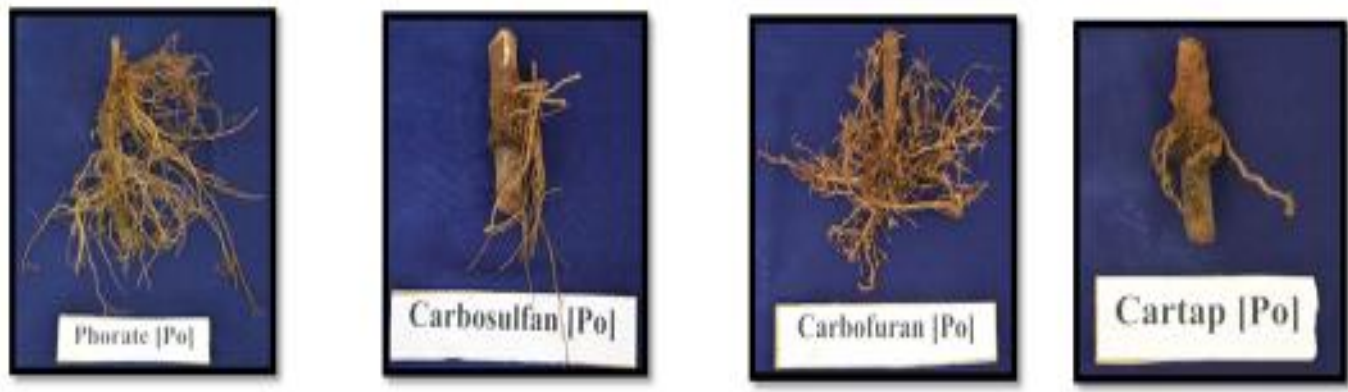

Application of nematicides one week after the inoculation of nematodes

Plate 4: Effect of nematicides on gall index and root growth parameter of pomegranate
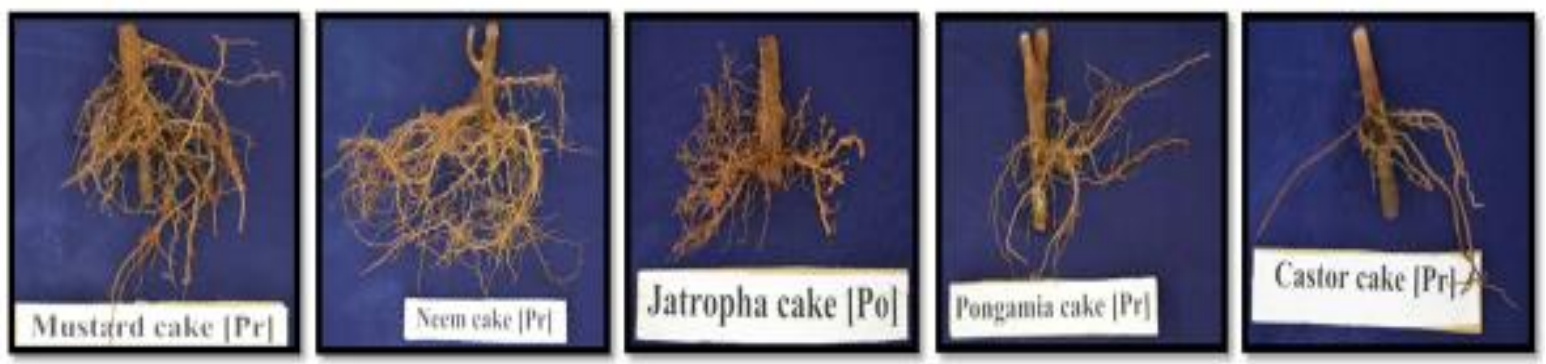

Application of oil cakes one week before the inoculation of nematode
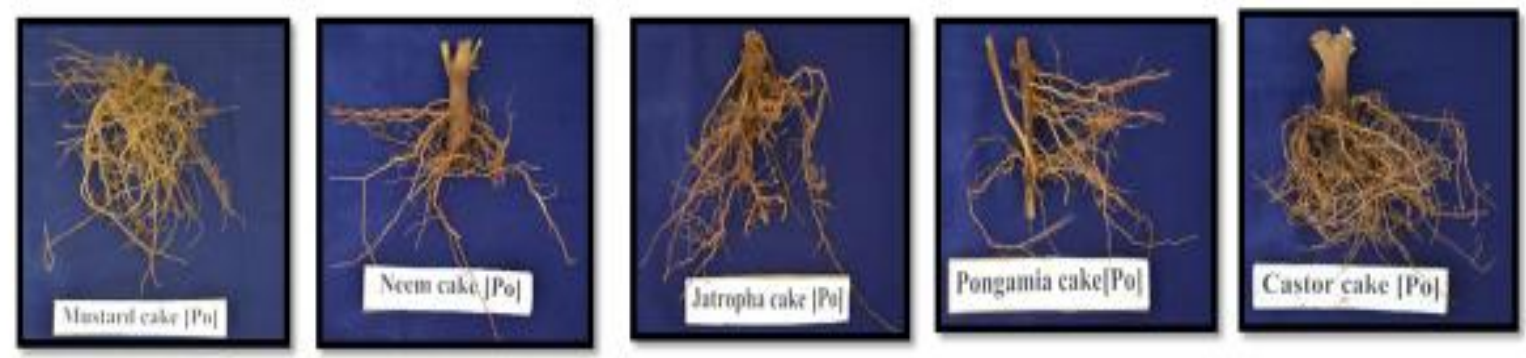

Application of oil cakes one week after the inoculation of nematode

Plate 5: Effect of oil cakes on gall index and root growth parameter of pomegranate 
Fig.1 Effect of nematicides on number of juveniles emerged

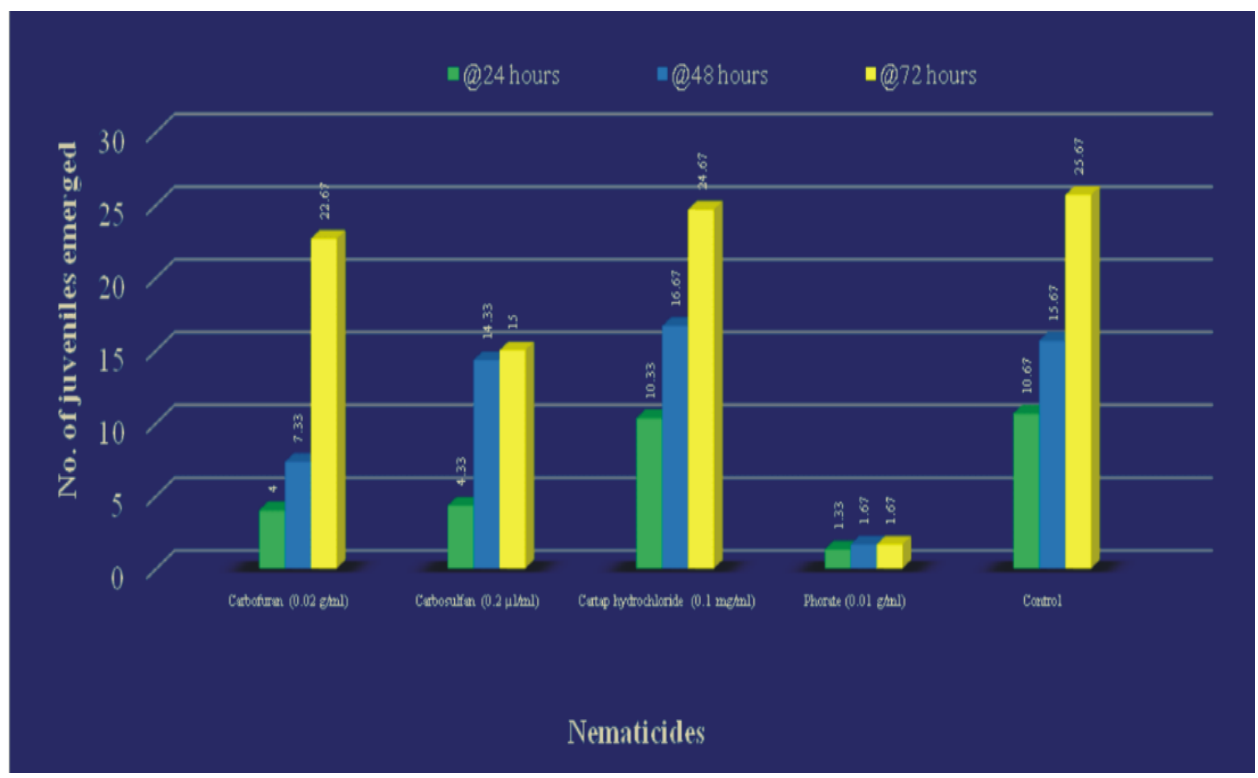

Fig.2 Effect of oil cakes on number of juveniles emerged

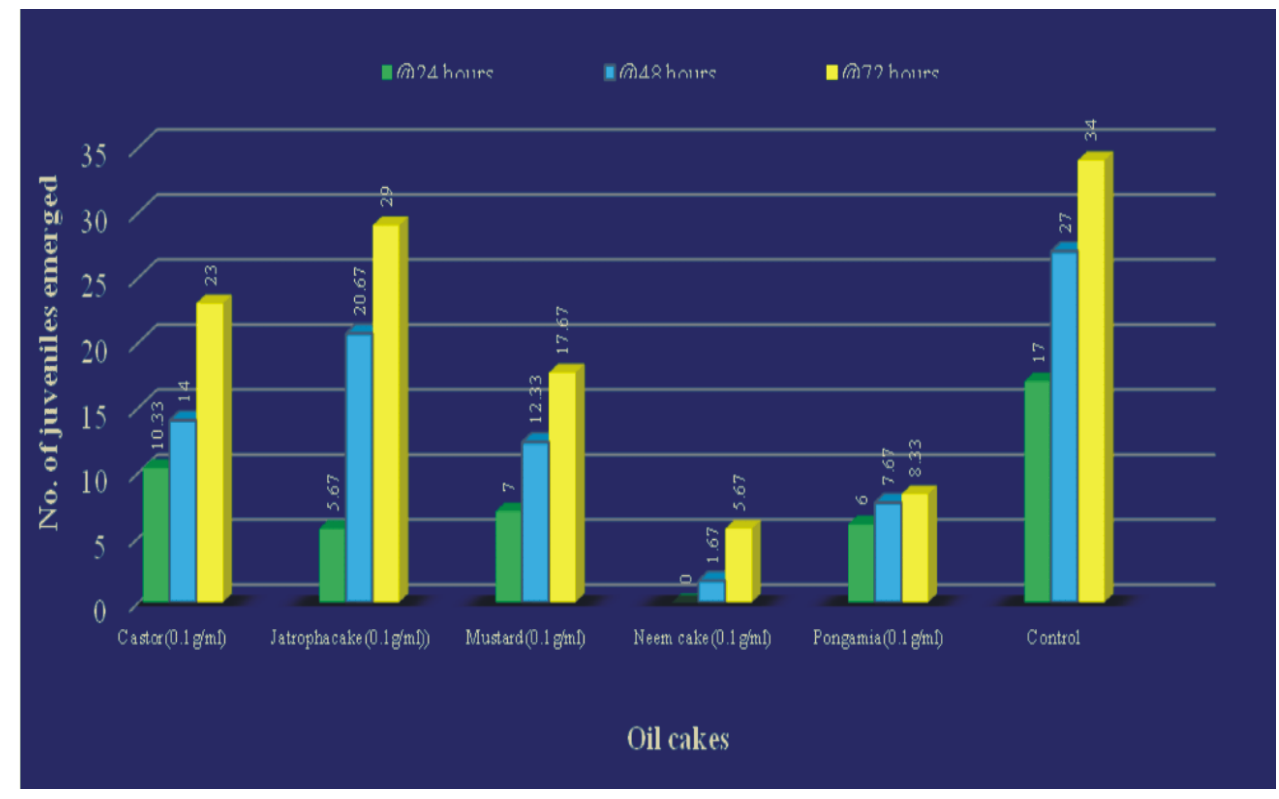


The chemical which effect on cholinergic (i.e., it mimics the action of neurotransmitter) system can have very dangerous effects which may lead to paralysis of the nematode. In a similar finding observed by Khan et al., 2012 where carbofuran and phorate through root dip plus single soil application provided greatest suppression in galling (16-20\%), egg mass production $(18-22 \%)$ and soil population (27.5-58.2\%) of M. graminicola. Mansoor (2006) reported neem leaf, neem seed powder, neem oil cake and two nematicides viz., carbofuran and phorate alone and in combination reduced the root knot development caused by Meloidogyne incognita in tomato. Highest reductions in the nematode infections and corresponding improvement in plant growth was noted in pots treated with neem oil cake combined with carbofuran.

The oil cakes were tested against number of juveniles emerged $M$. incognita in laboratory condition for their efficacy against the pathogen. Among the oil cakes neem cake was found significantly superior over all other nematicides in inhibiting the number of juveniles emerging capacity at 24, 48 and 72 hours followed by pongamia cake and mustard cake.

The specific chemical basis for the antinematicidal activity of neem remains obscure, although fractions containing steroids and terpenoid glycosides appear to be toxic in vitro to $M$. incognita (Akhtar, 2000). Nitrogen content of neem cake may also play significant role in reducing root knot nematode population in soil. Triterpene compounds in neem cake inhibit the nitrification process and provide more nitrogen in the form of ammonium to the plants for the same amount of nitrogen applied by the amendments (Akhtar and Alam, 1993). Therefore, application of oil cakes may be considered as the best option for root knot nematode not only because of its effectiveness and ease of availability, but also economic feasibility for the growers and environmental superiority.

Nematicidal properties of aqueous extracts of oil cakes or soil amended with oil cakes in the absence of plants have been proved to be challenging. Water soluble fractions of oil cakes extracted from neem, mahua, groundnut and castor were toxic to nematodes like Hoplolaimus indicus, Rotylenchulus reniformis and Tylenchorynchus brassicae and M. incognita (Khan et al., 1974). Similarly, the larval hatching of $M$. incognita was suppressed significantly by boiled extracts of mustard and cotton oil cake up to 99.92 and $99.38 \%$ in water. Eggs of $M$. incognita were found to be more vulnerable to oil cakes (neem, karanj, mahua, groundnut, cotton, linseed, sesamum and kokam) and fungicide (ceresan wet and aureofungin sol) treatment than larvae (Lanjeswar and Shukla, 1986). Neem cake extract was found to be most effective in killing $M$. incognita larvae (Gowda and Setty, 1978; Gowda and Gowda, 1999) whereas, mustard cake extract proved to be most effective in controlling Hoplolaimus indicus (Deshmukh and Prasad, 1969). However, greater concentrations of oil cake extract shown best results due to the presence of higher nematotoxic compounds.

Among the several methods of managing the plant diseases, soil amendment with organics is one of the effective methods. Amendments in the form of plant debris, green manures, farmyard manures, compost, oil cakes and fertilizers are known to improve crop productivity by improving nutrient status and soil tilth. Addition of amendments to soils might have increase microbial activities in soil to suppress diseases (Sivaprakasam, 1991).

Amendments of soil with decomposable organic matter is recognized as the most 
efficient method of changing soil and rhizosphere environment, thereby adversely affecting the life cycle of pathogens and enabling the plant to resist the attack of pathogens through better vigour or altered physiology. It was also reported that chemicals like ammonia (Khan et al., 1974) and fatty acids (Sitaramaiah and Singh, 1978) liberated during the decomposition of neem cake could be one of the factors involved in nematode control.

In the current study four nematicides and five oil cakes were tested against $M$. incognita in pots and checked for the effect ongall index andplant growth parameters Among the treatments, application of neem cake and phorate either prior or after inoculation of nematodes to pomegranate plants showed less gall index, high fresh shoot weight, dry shoot weight, fresh root weight, dry root weight, shoot length and root length. High shoot and root weight were also observed in castor cake treated plants but the number of galls was also more. The efficacy of mustered cake was low compared to neem cake and phorate in case of nematode management. The neem cake itself contains formaldehyde $(0.25 \%)$, which is responsible for nematode control (Sitaramaiah and Singh, 1978). Neem cake was more effective in reducing nematode population and improving tomato yield in pot experiments. Vijayalakshmi (2000) reported that aqueous extracts of neem seed and neem cake as root dip treatments were effective against Meloidogyne incognita infection in tomato. The castor cake though has showed good growth but it could not control nematode infection. The good growth may be nutrients supplied by the castor cake.

In conclusion, pomegranate (Punica granatum L.) is a fruit bearing deciduous shrub which has been rated as an important cash crop among horticulture crops. This crop is prone to several diseases, among which root knot caused by Meloidogyne incognita is a potential threat to successful crop production and their management is scanty. Hence looking to the economic importance of the crop, severity of the disease and future threat to the pomegranate cultivation, the present investigation was undertaken and results revealed that among the nematicides phorate@ 0.01 g/ml and neem cake @ 0.1 $\mathrm{g} / \mathrm{ml}$ were found effective over all other nematicides and oil cakes. It recorded significantly least numbers with respect to number of juveniles emerged. Among the treatments, neem cake and phorate applied plants before and after inoculation of $M$. incognita showed higher fresh shoot weight, dry shoot weight, fresh root weight, dry root weight, shoot length, root length and less number of galls.

\section{Acknowledgement}

I am thankful for giving me opportunity to work in this area to the Department of Plant Pathology, College of Horticulture, University of Horticultural Sciences, Bagalkot, 587104, Karnataka, India.

\section{References}

Akhtar, M., 2000. Nematicidal properties of neem tree Azadirachta indica. Integrated pest management. 5: 57-66.

Deshmukh, M.G. and S.K. Prasad., 1969. Effect of water soluble extracts of oilcakes on the population of Hoplolaimus indicus Sher. Indian J. Entom., 31: 273276.

Gowda, D.N. and K.G.H. Setty., 1978. Comparative efficacy of neem cake and methomyl on the growth of tomato and root-knot development. Curr. Res., 7: 118-120.

Gowda, P.D. and D.N. Gowda., 1999. In-vitro studies of oil-cake extracts on the survival of larvae of Meloidogyne 
incognita (Kofoid and White) Chitwood. Pl. Protec. Bull., 49: 10-11.

Jain, R. K. and Gupta, D. C., 1997. Efficacy of neem (Azadirachta indica) cake as nursery bed treatment in the management of root-knot nematode Meloidogyne javanica infesting tomato. Indian J. Nematol., 24: 241-251.

Khan, A., Alam, M. M. and Ahmed, R., 1974. Mechanism of the control of plant parasitic nematodes as a result of the applications of oil cakes to the soil. Indian J. Nematol., 4: 93-96.

Khan, M. R., Zaidi, B. and Haque, Z., 2012. Nematicides control rice root knot, caused by Meloidogyne graminicola. Phytopathol. Mediterr., $51 \quad$ (2): 298-306.

Khan, M.W., Alam, M.M., Khan, A.M. and S.K. Saxena, 1974. Effect of water soluble fractions of oil cake and bitter principles of neem on some fungi and nematodes. Acta Botanica Indica, 2: 120-128.

Lanjeswar, R. D. and V.N. Shukla., 1986.
Vulnerability of larvae and eggs of Meloidogyne incognita to some oil cakes and fungicides. Ind. J. Nematol., 16: 69-73.

Mansoor, A. S., 2006. Management of plant parasitic nematodes on tomato using neem products and nematicides. $J$. Ecofriendly Agric., 1(1): 73-74.

Sitaramaiah, K. and Singh, R. S., 1978. Effect of oil cakes on phenolic content of soil and plant and response of Meloidogyne incognita and its host related compound. Pl. Soil., 50: 671-679.

Sivaprakasam., 1991. Soil amendments for crop disease management. In: Basic Research for Crop Disease Management, Ed. Vidyasekaran, P., Daya Publishing House, New Delhi, pp. 382-404.

Vijayalakshmi, M., 2000. Effect of root dip treatments of tomato in aqueous extracts of neem products against, Meloidogyne incognita. Annu. Pl. Protec. Sci., 8: 272-273.

\section{How to cite this article:}

Madhushri Kerakalamatti, R. K. Mesta, K. C. Kiran Kumar and Rudresh, D. L. 2020. Evaluation of Nematicides and Oil Cakes against Root Knot Nematode caused by Meloidogyne incognita in Pomegranate. Int.J.Curr.Microbiol.App.Sci. 9(07): 1763-1775. doi: https://doi.org/10.20546/ijcmas.2020.907.202 